\title{
PRINCIPIOS DE JUSTIFICACIÓN EN LA RACIONALIDAD INFORMAL DE HILARY PUTMAN
}

Pablo Melogno *

\begin{abstract}
Resumen: En Razón, Verdad e Historia Hilary Putnam caracterizó la racionalidad informal como una alternativa que permite superar las limitaciones evidenciadas por las concepciones formalistas de la racionalidad. Se revisa inicialmente la caracterización ofrecida por Putnam y se establece que si bien Putnam niega todo principio universal de racionalidad, admite principios relativos, generales e indeterminados, que permanecen sujetos a necesidades de interrelación cognitiva con el entorno. A continuación, se muestra que los principios aceptan excepciones, y, a partir de aquí se defiende y analiza la posibilidad de revisión de principios cuya negación no resulta actualmente concebible. Finalmente, se afirma que tanto exponer como preservar creencias de la controversia constituyen elementos históricos que deben ser contemplados por una concepción informal de la racionalidad.
\end{abstract}

Palabras clave: Putnam, racionalidad informal, esquemas conceptuales, justificación

Abstract: In Reason, Truth and History, Hilary Putnam characterized informal rationality as an alternative that allows to overcome limitations evidenced by the formalist conceptions of rationality. Initially, the paper reviews the characterization provided by Putnam and establishes that, while Putnam denies any universal principle of rationality, he accepts relative, general and vague principles, that remain subject to needs of cognitive interrelationship with the environment. Secondly, we show that principles make exceptions and defend the possibility of reviewing principles whose denial is currently not conceivable. Finally, we state that both expressing and maintaining beliefs of controversy are historical elements that should be contemplated by an informal conception of rationality.

Keywords: Putnam, informal rationality, conceptual schemes, justification

\footnotetext{
${ }^{*}$ Prof. Adjunto de Epistemología e Historia de la Ciencia en la Escuela Universitaria de Bibliotecología y Ciencias Afines de la Universidad de la República, Montevideo, Uruguay. Se ha desempeñado como docente de Historia y Filosofía de la Ciencia en la Facultad de Humanidades y Ciencias de la Educación de la misma universidad, y como profesor de Lógica en el Instituto de Profesores Artigas. Dirección electrónica: pmelogno@gmail.com
} 


\section{Introducción}

A partir de su obra clásica de 1981, Razón, Verdad e Historia, ${ }_{1}^{1}$ y de trabajos como Las mil caras del realismo de 1987,2 Putnam desarrolló una concepción de la racionalidad que puede entenderse como una derivación de su defensa del realismo interno y como un intento por resolver algunos problemas de las teorías de la racionalidad de la primera mitad del siglo XX. Putnam caracteriza inicialmente la racionalidad informal como una modalidad alternativa a la racionalidad científica, que permite superar las limitaciones de las concepciones formalistas de la racionalidad. ${ }^{3}$ Defiende que no existen criterios universales para la justificación racional de creencias, sino sólo criterios de justificación dependientes del contexto y de los sistemas creencias a los que pertenecen. Esta noción de racionalidad informal no implica que la elección de criterios sea arbitraria, ni esté librada a la voluntad de quien pretende justificar una creencia. Implica más bien que la elección de los criterios, y por tanto la justificación de las creencias, están sujetas a necesidades de adaptación y de interrelación cognitiva con el entorno.

Una vez que responden a necesidades contextuales, las normas de aceptación racional de creencias operan en cuanto se institucionalizan en contextos específicos. Un ejemplo de ello es el desarrollo de la ciencia moderna, que muestra al método científico históricamente constituido por máximas y prescripciones que son seguidas por los científicos, y que explican buena parte del éxito de la ciencia. Pero se trata de máximas previas y ajenas a los componentes formales,

su aplicación requiere racionalidad informal, es decir, inteligencia y sentido común; no obstante, configuraron y configuran el conocimiento científico. En resumen, hay un método científico, pero este presupone nociones previas de racionalidad. No es un método que pueda servir como la parte más importante de la misma definición de la racionalidad. ${ }^{4}$

${ }^{1}$ Putnam, Hilary, Razón, verdad e historia, Tecnos, Madrid, 1988 (en delante RVH).

2 Putnam, Hilary, Las mil caras del realismo, Paidós/ I.C.E.-U.A.B., Barcelona, 1994 (en delante MCR).

${ }^{3}$ RVH, p. 186.

${ }^{4}$ Ibid., p. 193. 
A partir de esto, cabe indagar qué tipo de principios para la evaluación y justificación racional de las creencias pueden ser admitidos en el marco de una concepción informal de la racionalidad. Esto en cuanto Putnam ha negado que la evaluación racional de creencias pueda reducirse a principios algorítmicos, pero ha defendido que es posible la evaluación de creencias conforme a principios informales. En este sentido, si la racionalidad informal no admite los principios formales característicos de la racionalidad científica, cabe preguntarse qué tipo de enunciados pueden funcionar en ella a modo de principios.

\section{Racionalidad y necesidad cognitiva}

Putnam sostiene que los principios con que establecemos la racionalidad de nuestras creencias son revisables, en cuanto son producto de necesidades cognitivas derivadas de la interacción con el entorno. Explora las relaciones entre conocimiento y necesidad cognitiva revisando la premisa según la cual el que algo sea una creación humana lo vuelve arbitrario o no racionalmente elegible. Recurre para ello a un ejemplo de Ruth Anna Putnam. ${ }^{5}$ Señala R. A. Putnam que los cuchillos, como cualquier artefacto, son creados por el hombre, de acuerdo a sus necesidades y sin contar con un modelo pre-existente. Pero el que sean creaciones no impide que haya cuchillos mejores y peores, en cuanto evaluamos que son más o menos aptos en satisfacer las necesidades para las que fueron creados. Esto implica que en las elecciones acerca de fines y valores, no es necesario que éstos sean trascendentes o pre-existentes para asegurar la racionalidad y el contenido cognitivo; del mismo modo que aceptar que los fines y valores son creaciones humanas no implica que no pueda haber deliberación racional respecto de que fines adoptar. Es por esto que la evaluación de las creaciones humanas -éticas, artefactuales o teóricas- remite al concepto de necesidad:

Es porque hay necesidades humanas reales, y no simplemente deseos, por lo que tiene sentido distinguir entre valores mejores y peores, y, por lo

${ }^{5}$ Putnam, Ruth Anna, "Creating Facts and Creating Values", Philosopby, Vol. 60, N 232, 1985, pp. 187-204. 
mismo, entre cuchillos mejores y peores (...) que las necesidades humanas tampoco pre-existen, que la humanidad está constantemente rediseñándose a sí misma, y que nosotros creamos las necesidades. ${ }^{6}$

A partir de aquí puede pensarse que si dos sujetos poseen esquemas conceptuales incompatibles, esto responde a que tienen necesidades y propósitos cognitivos diferentes, y en esa medida es posible afirmar que las elecciones de ambos son racionales. Desde este modo, la noción de racionalidad informal involucra una perspectiva realista que reconoce la diversidad de contextos cognitivos y la conformación de diferentes esquemas conceptuales a partir de ellos. Existen hechos exteriores a los esquemas, pero sólo podemos saber algo de ellos una vez que hemos elegido un esquema para conceptualizarlos. ${ }^{7} \mathrm{La}$ exterioridad de los hechos permite explicar que respondan mejor a unos esquemas que a otros, así como también que la adopción de un esquema conceptual responde a que aumenta nuestra capacidad explicativa, nos provee mejores predicciones, o satisface algún otro criterio epistémico. Pero, para una caracterización de la racionalidad como la defendida por Putnam, es central que en la codependencia entre principios y experiencia sea el mundo empírico el que provee de razones para modificar los principios de aceptabilidad racional. Por esto, cabe pensar que una revisión de principios es racional si es resultado de un cambio de nuestras necesidades en relación a la experiencia, y si tiene como objetivo reajustar nuestro sistema de creencias al mundo empírico, y no es racional si no responde a una modificación significativa de la experiencia.

\section{Principios de racionalidad}

En MCR Putnam afirma que quien pretende que sus creencias son racionales, y a la vez las protege de la crítica racional, está incurriendo en una contradicción 8 . De esto podría extraerse como principio de racionalidad: no es racional sustraer a la crítica las creencias que se postulan como racionales. Si bien Putnam introduce estas consideraciones en un contexto

${ }^{6}$ MCR, pp. 150-151.

7 Ibid., pp. 86-87.

${ }^{8}$ Ibid., p. 114. 
polémico, cabe pensar que un principio así formulado es coherente con la racionalidad informal. En primer término, el enunciado establece que no es racional sustraer ciertas creencias a la crítica, pero no cuáles son las condiciones de la crítica, ni las condiciones por las que una creencia podría llegar a postularse como racional. Esto significa que puede haber sujetos con diferentes esquemas conceptuales que involucren diferentes nociones de racionalidad, y puede decirse de cada uno que su actitud no será racional si sustrae alguna creencia que pretende sea racional a los códigos de racionalidad de su propio esquema.

En vistas de esto puede decirse que se trata de un principio general pero indeterminado, en cuanto sólo adquiere contenido al asociarse a pautas de racionalidad concretas provenientes de un esquema conceptual. Se trata de una generalidad análoga a la que Putnam atribuye al enunciado la ciencia busca la verdad, ya que éste, tomado como un principio de definición de los fines de la ciencia, no adquiere contenido hasta que se asocia a parámetros de aceptabilidad bajo los que los científicos llevan a cabo sus investigaciones. El contenido material del enunciado involucra pautas de aceptabilidad racional, acerca de qué es la objetividad, qué es una investigación racional o qué constituye una buena razón y que no. ${ }^{9} \mathrm{Y}$ siendo estas pautas históricamente variables y relativas a cada esquema, un enunciado de este tipo sólo establecería una clase de procedimiento para la justificación racional, pero no un algoritmo independiente de los contextos de aplicación.

Frente a esto, cabe señalar que en la década de los 70’s Putnam había desarrollado una extensa discusión sobre las proposiciones a priori, en vistas de establecer la posibilidad de afirmar algún principio absolutamente a priori, y por tanto, racionalmente elegible en toda circunstancia. En $R V H$, mientras tanto, señala que criterios como la simplicidad o la utilidad pueden llevar a abandonar razonablemente un principio asumido como a priori. Contra Quine,$^{10}$ insiste en que hay enunciados no-revisables, y se pregunta bajo qué condiciones podría aceptarse un enunciado como "todos los enunciados son

\section{${ }^{9} \mathrm{R} V H$, p. 132.}

10 Quine, Willard Van Orman, "Dos dogmas del empirismo", en Desde un punto de vista lógico, Orbis, Buenos Aires, 1984, pp. 49-81. 
verdaderos", 11 aunque en último término limita el alcance de los principios a priori a algunos casos básicos. ${ }^{12}$ Sin embargo, puede pensarse que a nivel metodológico existen diferencias entre (1) no todos los enunciados son verdaderos, y (2) no es racional sustraer a la crítica los enunciados que se consideran racionales, en cuanto a efectos de la justificación racional, (2) tiene más alcance y fertilidad que (1). Supóngase un sujeto $S$ que debe optar entre las creencias $C 1$ y $C 2$. Supóngase que $C 1$ es la negación de C2. En este caso la aplicación de (1) sólo le permite a $S$ saber que no puede elegir al mismo tiempo $C 1$ y $C 2$, y acaso le permitiría inferir que si las consecuencias de una de las creencias son verdaderas no pueden serlo las consecuencias de la creencia rival, pero (1) no le proporcionaría a $S$ más elementos que estos para comparar $C 1$ y $C 2$, por lo que no le permitiría establecer qué creencia es más racional. Sin embargo, si $S$ aplica (2), obtiene que $C 1$ y $C 2$ deben ser evaluadas en igualdad de condiciones, en cuanto ninguna de las dos puede racionalmente- sustraerse a la crítica efectuada conforme a los parámetros de racionalidad que $S$ acepta. En un marco de racionalidad informal, no es seguro que por este procedimiento derivado de (2) $S$ logre determinar qué creencia es más racional, ya que si esto sucediera (2) tendría el status de un principio algorítmico. Pero sí es posible que a través de (2) $S$ logre establecer cuál de las dos creencias es más racional, condición que no se cumple para (1).

Una concepción informal supone también aceptar que otro sujeto $S$ ' proponga evaluar $C 1$ y $C 2$ en función de un principio (3) diferente de (1) y (2). En este caso, la adecuación de (3) también podría ser evaluada, comparando (3) con (2), de la misma forma que (2) fue comparado con (1). Si el contenido de (3) fuera es racional no dar motivos de por qué se considera racional una creencia, podría decirse justificadamente que (3) no es aceptable

${ }^{11}$ RVH, p. 90.

12 Estas consideraciones que aparecen algo sintetizadas en $\mathrm{R} V H$ constituyen el corolario de la discusión emprendida por Putnam en torno a las proposiciones a priori. En "There is at least one a priori truth", llama principio minimo de no-contradicción al enunciado no toda proposición es verdadera y falsa, efectuando una defensa de su carácter a priori; Putnam, Hilary, "There is at least one a priori truth", en Realism and Reason, Philosopbical Papers, Cambridge University Press, Cambridge/ Mass, 1983, Vol. 3, pp. 98-114. 
como principio de racionalidad. ${ }^{13}$ En esta medida, el que los principios sean revisables no conduce a que no puedan compararse, ni a que no pueda decidirse con buenas razones qué principio adoptar en cada caso.

Sin embargo, puede haber casos más problemáticos que la comparación de (3) con (2), y que funcionan como restricciones de la generalidad de (2). Puede suponerse un sujeto S", defensor de $C 1$ y renuente a aceptar C2, que proponga el principio (4): Bajo ciertas condiciones es racional sustraer algunas creencias a la crítica, con lo que quedaría abierta la posibilidad de que $S$ opte por $C 1$ y $S$ " por $C 2$. Es posible que en este caso no haya forma de establecer que la elección de $S$ es más racional que la de $S$ " o viceversa, en cuanto es concebible que (4) resulte para el esquema conceptual de $S$ " tan metodológicamente adecuado como (2) resulta para $S$. Principios como (2) resultan altamente funcionales como principios de racionalidad, pero conforme a los términos de la racionalidad informal, se trata de principios indeterminados y de generalidad restringida, en cuanto no establecen condiciones específicas de aplicación y en cuanto admiten legítimamente restricciones derivadas de la interposición de otros principios.

En la contraposición de (2) con (4) se configura el escenario básico de una concepción informal de la racionalidad: dos sujetos optan por creencias opuestas resultando racionales las elecciones de ambos. Esto no implica que los principios no sean comparables, implica que no existe algoritmo de comparación, y que en algunos casos las comparaciones son posibles y en otros no. Lo que resulta fundamental en una concepción informal es la exclusión de dos escenarios: la posibilidad de decidir de modo concluyente cuál de los principios en juego es el adecuado, y la imposibilidad de tomar cualquier decisión sobre su adecuación; pero lo que no se excluye es la existencia de principios de racionalidad ni de mecanismos de comparación y evaluación.

${ }^{13}$ Se podría alegar que (3) es autocontradictorio, aduciendo que la racionalidad se basa en dar razones de lo que se cree o lo que se hace. Pero si quien interpone (3) insiste en que se puede ser racional sin dar razones, debería proporcionar razones para aceptar su concepción de la racionalidad, o insistir en que su concepción de la racionalidad sea aceptada sin mediar razones. En el primer caso está siendo incoherente con (3); en el segundo caso no lo está siendo, pero debilita su posición en cuanto no proporciona ningún elemento para aceptar (3). 
A pesar de esto, no deja de ser llamativo que Putnam afirme en $M C R$ el principio (2) no es racional sustraer a crítica los enunciados que se consideran racionales, mientras que en $\mathrm{R} V H$ rechaza el principio quineano según el que ningún enunciado es inmune a la revisión (5). Frente al parentesco que presentan ambos principios, cabe preguntarse si es posible aceptar el primero y rechazar el segundo. Esto debería ser posible si la postura de Putnam entre RVH y MCR fuera consecuente, mientras que si aceptar (2) implica aceptar (5) cabría pensar que en $M C R$ Putnam habría reformulado parcialmente su concepción de la racionalidad para dar cabida a principios rechazados en $\mathrm{R} V H$. Un argumento que se puede ofrecer tiene que ver con el ámbito de aplicación: (5) establece que no hay enunciados inmunes a la revisión, por lo que no es racional sustraer a la crítica ningún enunciado, y (2) establece que no es racional sustraer a la crítica solamente los enunciados que se han declarado racionales. Un sujeto podría sustraer a la crítica una o más creencias correspondientes a su esquema conceptual por el simple de no conferirles un status racional, y de esta forma estaría aceptando (2) y no (5), ya que de acuerdo a (5) esta posibilidad queda vedada, en cuanto el sujeto estaría obligado a someter a revisión cualquier creencia que forme parte de su sistema.

Hasta aquí resulta consistente que Putnam haya negado (5) y afirmado (2), sin embargo la cuestión presenta aún alguna dificultad. Putnam presentó en $R V H$ (1) como caso contrario a (5), en cuanto no parece plausible pensar en condiciones bajo las que sea lícito aceptar que todos los enunciados son verdaderos. Pero si se acepta que todos los enunciados racionales son pasibles de revisión (2), parece necesario aceptar que un enunciado como (1) es pasible de revisión, salvo que se quiera tomar la alternativa poco prometedora de excluirlo del dominio de la racionalidad. Si bien lo afirmado por Putnam en $M C R$ no conduce automáticamente a la aceptación del principio de Quine, puede sí decirse que la introducción del principio (2) en $M C R$ implica una reformulación parcial de la caracterización de la racionalidad introducida en $R V H$; reformulación que deja más espacio a la revisión de principios y menos margen para la defensa de elementos a priori.

¿Pero bajo qué condiciones podría ser revisable la afirmación de que no todos los enunciados son verdaderos? Quizás la forma de compatibilizar (1) con (2), o de incluir (1) en el radio de acción de (2) -a saber el de los enunciados revisables-, no consiste en especificar bajo qué condiciones 
estaríamos dispuestos a revisar nuestra creencia de que no todos los enunciados son verdaderos, sino en aceptar que en cuanto la consideramos una creencia racional, estaríamos dispuestos a revisarla si se dieran las condiciones que justificaran hacerlo, aun cuando actualmente no estemos en condiciones de especificarlas. Podemos considerar (1) como un principio fundamental para nuestras pautas de racionalidad, ya que no concebimos condiciones en las que pueda ser racionalmente negado, pero podemos al mismo tiempo establecer que estaríamos dispuestos a revisarlo si se dieran las condiciones adecuadas. Hasta aquí, el cumplimiento de (2) parece requerir antes la disposición a la revisión que la declaración de las condiciones de posibilidad de la revisión; por el contrario, si se pretende concederle un carácter estrictamente a priori, es necesario negar o moderar (2). ${ }^{14}$

\section{Consenso y controversia}

La noción de racionalidad informal habilita que, en la comparación entre los principios (2) y (4), dos sujetos defiendan creencias opuestas, y que tanto la elección de uno como de otro sea racional. Es así como quedan incluidos en la racionalidad los casos en que no hay una posibilidad de

14 Putnam había defendido que el principio minimo de no-contradicción es absolutamente a priori o irrevisable en términos epistémicos. Entre otros argumentos, señalaba que si toda proposición es revisable bajo ciertas circunstancias, habría circunstancias bajo las que sería racional revisar el principio minimo de no contradicción. Pero al no haber tales circunstancias, al no poder dar cuenta de ellas, entonces se trata de una proposición que no tiene circunstancias de revisión racional. Aquí resulta problemático el tránsito de no podemos concebir circunstancias que justifiquen la revisión racional a no bay circunstancias que justifiquen la revisión racional; lo que parece tratarse de un desplazamiento -no justificado- de lo que Putnam llama contextualmente a priori a lo que denomina absolutamente a priori. Véase Putnam, Hilary, "There is at least one a priori truth", ed. cit., pp. 99-101. Finalmente afirma que a efectos de la mayoría de los propósitos relevantes de la argumentación filosófica, basta con que podamos identificar una proposición cuyas circunstancias de negación no sean actualmente inconcebibles, dado lo dificultoso de mostrar que son inconcebibles en absoluto: "If a statement has the property that we cannot now describe any circumstances under which it would be rational to give it up, that will surely suffice for most for most purposes of philosophical argument" (Ibid, p. 114). 
fundamentación última que permita establecer cuál de los principios en conflicto es más racional o está mejor justificado. Es claro que para Putnam estos casos no implican una insuficiencia de su concepción de la racionalidad, en cuanto permiten mostrar que la elección racional no es suficiente para la supresión del conflicto, y que la racionalidad no equivale al consenso.

Profundizando este punto, puede tomarse el cuestionamiento por Putnam del status de la racionalidad científica como base para afirmar que la racionalidad no implica la ausencia de desacuerdo. Weber había establecido que no es posible determinar la verdad de un juicio de valor para toda persona racional, mientras que sí es posible lograrlo en la ciencia. ${ }^{15} \mathrm{El}$ carácter público del conocimiento científico, y la revisión pública de resultados permitiría alcanzar niveles de asentimiento racional que no admiten las cuestiones de valor. Putnam considera el argumento de Weber como un argumento mayoritarista, señalando un problema de circularidad en la definición de lo racional. Si cuando detectamos una teoría científica que cuenta con apoyo mayoritario, definimos los procedimientos racionales como aquellos que permiten el asentimiento de la mayoría, tendríamos que definir la falta de unanimidad en ética como la imposibilidad de lograr un acuerdo mediante los métodos que provocan el asentimiento de la mayoría. Esto implica que primero percibimos que los juicios de valor no pueden ser asentidos de igual manera por la mayoría de las personas, y luego declaramos que la falta del asentimiento es un indicador de no racionalidad. Para Putnam esto no es una prueba de la racionalidad, sino una identificación no justificada del asentimiento de la mayoría con el asentimiento racional ${ }^{16}$.

Las posturas mayoritaristas parten de que es posible alcanzar un alto nivel de consenso en las teorías científicas, lo que para Putnam tampoco es aceptable. Lejos del asentimiento racional, la mayoría de los legos aceptan las teorías científicas porque permiten generar tecnología funcional o porque confían en los expertos. Pero el lenguaje especializado de las teorías científicas las vuelve incomprensibles para la mayoría, ${ }^{17}$ por lo que los

15 Weber, Max, "La objetividad cognitiva de la ciencia social y de la política social", en Ensayos sobre metodología sociológica, Amorrortu, Buenos Aires, 1990, pp. 39-101.

${ }^{16} \mathrm{RVH}$, pp. 177-179.

17 Ibid., p. 178. 
niveles reales de consenso son bastante menores de lo que supondría Weber. Señala asimismo Putnam que si sólo se considera conocimiento objetivo lo que está estrictamente más allá de toda controversia, entonces muy poco de la ciencia presente se puede considerar conocimiento, en cuanto difícilmente pueda decirse que algún sector de la ciencia está despejado de toda controversia. ${ }^{18}$ Estas consideraciones tienen un vínculo muy estrecho con la concepción falibilista del conocimiento defendida por Putnam, no obstante presentan algunas aristas que merecen una revisión detenida.

En esta parte de su argumentación, Putnam no parece distinguir del todo entre creencias que están más allá de toda controversia en condiciones epistémicas ideales ${ }^{19}$ y creencias que son consideradas más allá de toda controversia en condiciones históricas reales ${ }^{20}$. Una revisión histórica puede inducir a pensar que no hay nada más allá de toda controversia, en cuanto creencias que en un momento se consideraron incontroversiales posteriormente fueron abandonadas. Pero la misma revisión mostraría que los seres humanos tienden a asumir creencias como incontroversiales, con cierta independencia de los antecedentes históricos. Para una concepción falibilista del conocimiento no hay ninguna creencia que esté exenta de controversia; pero aún cuando sean aceptadas las premisas del falibilismo, desde un punto de vista histórico parece necesario conceder que durante varios períodos de la historia ha habido creencias que se han considerado verdaderas más allá de toda duda.

${ }^{18}$ MCR, 125-126.

${ }^{19} \mathrm{R} V \mathrm{H}, 65$.

20 Putnam había explicitado la distinción algunos años antes, en la formulación del concepto de aprioridad contextual. Señala que es necesario aceptar que a lo largo de la historia hay teorías que han sido asumidas como verdaderas más allá de toda duda. Se trata de teorías que sólo pueden ser revisadas a la luz de una teoría rival (que en muchos casos las terminará derrocando), y que pueden permanecer durante largos períodos asumidas como verdaderas más allá de toda duda, en cuanto quienes las sostienen no conciben condiciones bajo las que puedan ser negadas o expuestas a revisión. Putnam entendía que este tipo de teorías tienen un status contextualmente a priori, en cuanto bajo condiciones históricas concretas ofician como verdades que no están sujetas a revisión. Véase Putnam, Hilary, "Two dogmas revisited", en Realism and Reason, Pbilosophical Papers, Cambridge University Press, Cambridge/ Mass, 1976, Vol. 3, pp. 87-97. 
Frente a esto podría pensarse que tenemos razones filosóficas de fondo para comprometernos con el falibilismo, aceptando que toda creencia está sujeta a controversia, y que quienes a lo largo de la historia han tomado algunas creencias como no sujetas a controversia han incurrido en una actitud errónea o irracional. Pero esta estrategia no parece constituir una buena de defensa de la racionalidad informal, ya que ${ }^{21}$ supondría colocarse en lo que Putnam ha denominado el punto de vista del espectador, ${ }^{22}$ en cuanto estaríamos otorgando a la defensa filosófica del falibilismo un lugar más allá de las condiciones en que los seres humanos sostienen efectivamente sus creencias. En contraposición, desde lo que Putnam llama el punto de vista del agente parece necesario admitir que si bien un aspecto fundamental del hecho de sostener creencias pasa por exponer algunas de ellas a controversia, otro aspecto no menos importante pasa por considerar otras como exentas de controversia, siendo ambos elementos históricos que deben ser contemplados por una concepción informal de la racionalidad. Esta dificultad puede despejarse recurriendo a una distinción introducida por Putnam en la década de los 70's, entre proposiciones irrevisables desde el punto de vista conductual y proposiciones irrevisables desde el punto de vista epistémico. Una proposición irrevisable conductualmente es una proposición que -bajo condiciones históricas reales- no estamos dispuestos a abandonar; por lo que en este caso la irrevisabilidad es una constatación acerca de nuestra conducta cognitiva. Una proposición irrevisable epistémicamente, mientras tanto, es una proposición que nunca sería racional abandonar ${ }^{23}$, no aludiendo la irrevisabilidad a una descripción de nuestra conducta sino a una especificación del status de la proposición.

Así, podemos admitir que en la ciencia contemporánea existen proposiciones actualmente irrevisables, es decir, conductualmente irrevisables, sin que ello implique que sean epistémicamente irrevisables. Esto es, una proposición puede ser irrevisable en la medida en que actualmente no concebimos las condiciones bajo las que podría ser expuesta a la controversia, lo que no la convierte sin embargo en epistémicamente irrevisable, en cuanto las condiciones podrían darse en el futuro como

${ }^{21}$ Además de las razones ya aludidas en relación a la verdad como aceptabilidad racional bajo condiciones epistémicas ideales.

${ }^{22}$ MCR, 137, 146-147.

${ }^{23}$ Putnam, Hilary, "There is at least one a priori truth", ed. cit., p. 98. 
consecuencia de la irrupción de nuevos conocimientos. Esto no implica una puesta en riesgo del falibilismo, en cuanto podemos insistir en que tenemos buenas razones para considerar revisables la mayoría de nuestras creencias, y aceptar que otros sujetos en contextos diferentes al nuestro tuvieron buenas razones para considerar como no revisables algunas de las suyas. Difícilmente pensaríamos hoy en día que la física de Aristóteles es una teoría no sujeta a controversia, pero podemos afirmar que durante la Edad Media fue racional la actitud de quienes la consideraron irrevisable. A efectos de una caracterización adecuada de la racionalidad, parece necesario distinguir entre el punto de vista filosófico según el que no cabe considerar ninguna creencia como más allá de toda controversia en términos absolutos ${ }^{24}$, y la constatación histórica de que en diversas épocas hay creencias que han sido asumidas como no sujetas a controversia.

\section{Conclusión}

El tratamiento de los problemas de la racionalidad por parte de Putnam no arroja, como muchos otros aspectos de su obra, una imagen única y sistemáticamente ensamblada, sino más bien una compleja serie de reformulaciones, matices y hasta eventuales contradicciones que se van desplegando a medida que Putnam profundiza el análisis. Estos virajes y reorientaciones de pensamiento tan característicos de la filosofía de Putnam son parte de lo que lo convierte en un autor tan estimulante para el debate filosófico, al tiempo que ofician como motivación para esfuerzos de análisis y reconstrucción como el que aquí se ha presentado.

La caracterización de la racionalidad ofrecida por Putnam conlleva la negación de principios universales y algorítmicos para la justificación racional de creencias, pero no impide la defensa de otra clase de principios de racionalidad. La racionalidad informal admite principios relativos, en la medida en que son dependientes de necesidades cognitivas históricamente variables, y permanecen asociados a esquemas conceptuales. Constituyen principios indeterminados, en cuanto no establecen contenidos ni condiciones concretas de aplicación, y de generalidad restringida, en cuanto son revisables a la luz de principios rivales igualmente fértiles o adecuados.

${ }^{24}$ Putnam, Hilary, “Two dogmas revisited”, ed. cit., p. 95. 
Esto supone que una vez que la revisión de los principios es amplia pero no ilimitada, resulta posible evaluar la adecuación y la fertilidad de principios en conflicto, si bien el resultado de la evaluación no es necesariamente unívoco ni concluyente. En estos términos, la posibilidad de revisión racional puede ampliarse hasta abarcar principios cuyas condiciones de revisión no resultan actualmente concebibles.

De acuerdo a esta caracterización de racionalidad, la elección racional no implica la supresión de la controversia, por lo que la concepción de Putnam permite ver que resulta inconducente considerar que sólo pueden tildarse de racionales las creencias establecidas más allá de toda controversia. Sin embargo, al afirmar esto, Putnam no parece ubicarse del todo entre creencias que están más allá de toda controversia en condiciones epistémicas ideales, y creencias que son consideradas más allá de toda controversia en condiciones históricas reales. Sobre esta base, cabe pensar que un aspecto fundamental de tener creencias pasa por exponer algunas de ellas a controversia, y otro aspecto no menos importante pasa por considerar otras como exentas de controversia, siendo ambos elementos históricos los que deben ser contemplados por una concepción informal de la racionalidad.

Recibido: 03/2012. Aceptado: 08/2012 